\title{
THE SUCCESSFUL REMOVAL OF A TUMOUR FROM THE RIGHT ATRIUM UNDER HYPOTHERMIA
}

\author{
BY \\ M. KRCIÍLKOVÁ, J. MUSIL, J. NAVRÁTIL, AND O. OLEJNÍK \\ From the Department of Surgery, School of Medicine, University of Brno, Czechoslovakia
}

(RECEIVED FOR PUBLICATION MAY 1, 1958)

Primary tumours of the heart are rare, and most of those reported in the literature have been histopathological observations from the postmortem table. It is to be expected that in future cardiac tumours will be diagnosed more frequently during the patient's life and before operation, thanks to diagnostic improvements in cardiology and radiography. It is equally to be expected that the number of tumours successfully removed from the heart will increase if an operative technique on the open heart under hypothermia or extracorporeal circulation is used (Bickford, Egan, and Bryce, 1956). To achieve this success more experience must be collected, and that is why we report our patient in whom the cardiac tumour was diagnosed before operation and successfully removed from the open heart under hypothermia.

\section{CASE RePORT}

D. S., a thin, tall girl, 14 years of age, had had frequent tonsillitis during her early childhood, so that at the age of 2 years tonsillectomy had to be performed. Till the age of 6 she was without any complaints and took part in games suitable to her age. At this age, after measles, she began to have precordial pain. She then spent some time in hospital and was treated for rheumatic carditis, after which she remained under ambulatory control. At the age of 10 hepatomegaly was noticed. Between 12 and 13 years she was once more admitted to hospital because of dyspnoea and an elevated blood sedimentation rate. In the latter half of the year before operation she was treated for increasing shortness of breath, complaints of weakness, palpitations, and cyanosis.

She was admitted to our clinic on July 8, 1957, and was hardly able to undertake even the least physical exertion because of dyspnoea.

At physical examination a striking mitral facies and gross systolic distension of the jugular veins were noticed. There was a slight prominence of the chest in the precordial region and marked systolic pulsation of the right ventricle. The liver was enlarged, and the edge was palpable four fingerbreadths below the right costal margin also with marked systolic pulsation. The heart sounds were indistinct and a systolic murmur was heard in the fifth intercostal space, loudest near to the right sternal border. The murmur changed its character and intensity according to the position of the patient. The blood pressure was $115 / 80 \mathrm{~mm}$. $\mathrm{Hg}$; the pulse was regular and easily palpable in all extremities.

The electrocardiographic tracing (Fig. 1) showed right bundle-branch block and right ventricular overload.

The postero-anterior chest radiograph (Fig. 2) revealed small hila and clear lung fields. At the same time there was enlargement of the right ventricle with expansile systolic pulsation of the right atrium. By contrast the pulmonary artery and aorta were small. Both the venae cavae were enlarged and showed intense distension during systole. On fluoroscopy a dense, well-circumscribed, ball-shaped. and plum-sized mass could easily be seen within the shadow of the right atrium, following the systolic and diastolic movement of the right heart in a quick, sometimes irregular, swinging curve. At the beginning of right ventricular contraction this mass was shot away from the tricuspid valve in the direction of the orifice of the inferior vena cava. At the onset of diastole it moved back again to the tricuspid orifice. This swinging mass could well be seen in all radiographic projections. From the curve of its pathway one could presume that it was fixed by a stalk somewhere in the atrium near the orifice of the inferior vena cava. It was impossible to snap the moving mass on a kymogram.

The angiocardiogram demonstrated a defect in the contrast medium filling the right atrium and corresponding to the ball-like mass (Fig. 3).

These clinical findings resulted in the diagnosis of right atrial polypoid tumour, with tricuspid valve insufficiency.

\section{OPERATION}

After previous " neurovegetative blockades," three days before the intervention, on July 19, 1957, operation under hypothermia at a lowest temperature of $27^{\circ} \mathrm{C}$. was performed.

In the prone position a transverse sternum-splitting incision was made in the third left and fourth right 
FIG. 1. - Electrocardiogram with typical changes of right bundle-branch block and overloading of the right ventricle.

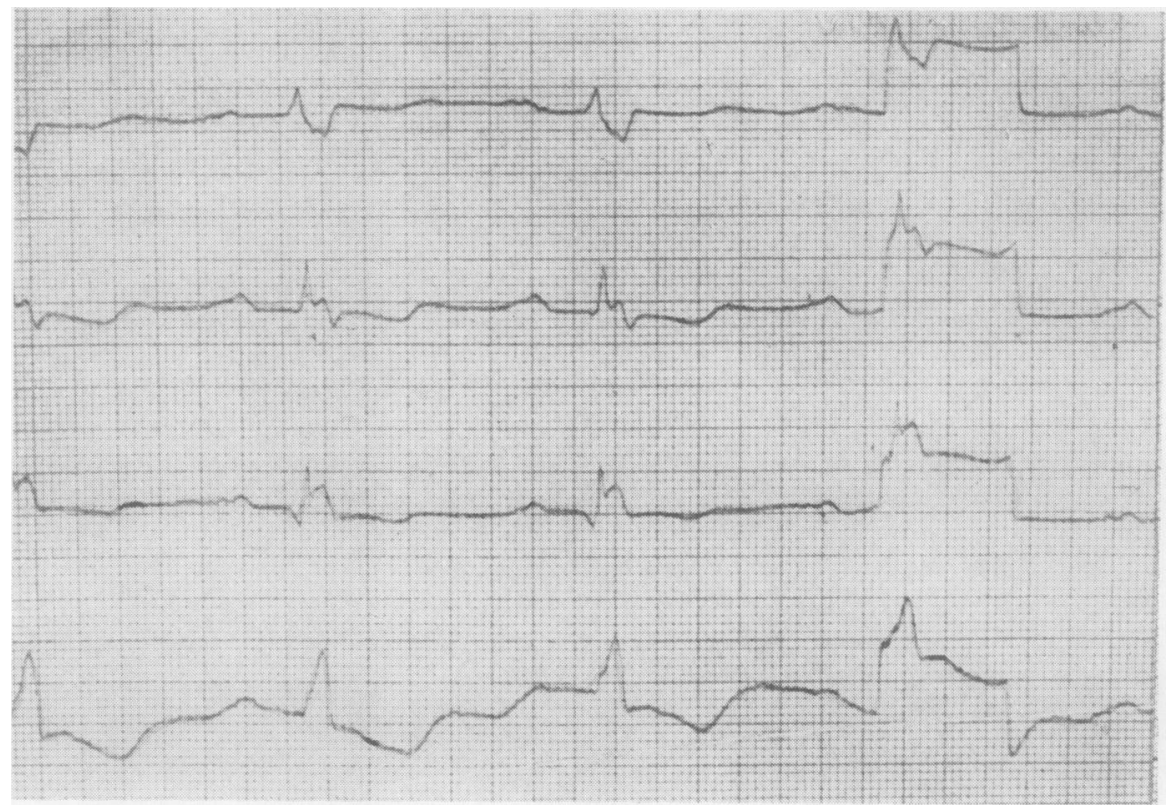

intercostal spaces. The right atrium and auricle were immense. The wall of the atrium was dense and thick and covered by fibrinous deposits. The aorta and pulmonary artery were hypoplastic and about $2 \mathrm{~cm}$. in diameter. On intracardiac investigation through the right auricle a ball-shaped tumour about the size of a peach and hanging on a stalk could be palpated. The stalk of the tumour was about $6 \mathrm{~mm}$. in diameter and $1.5 \mathrm{~cm}$. in length and allowed swinging movements between the orifice of the inferior vena cava and the tricuspid ring, which was enlarged.

The cusps of the tricuspid valve were normal.

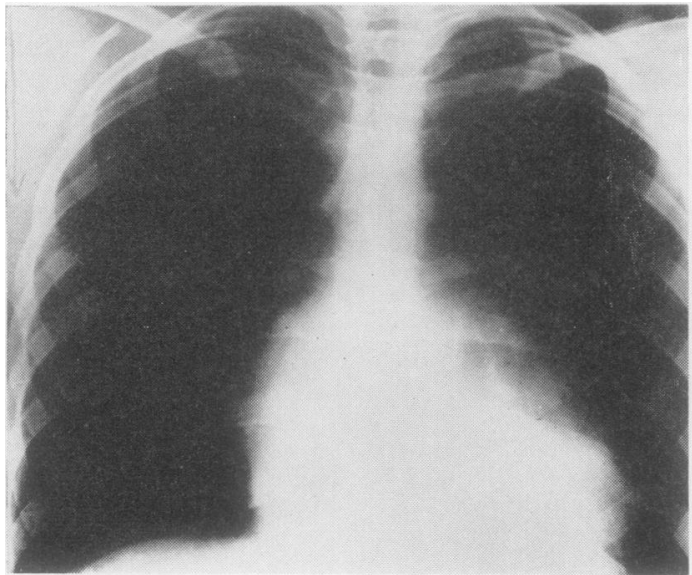

Fig.2.-Postero-anterior view of the chest showing right heart enlargement pre-operatively.

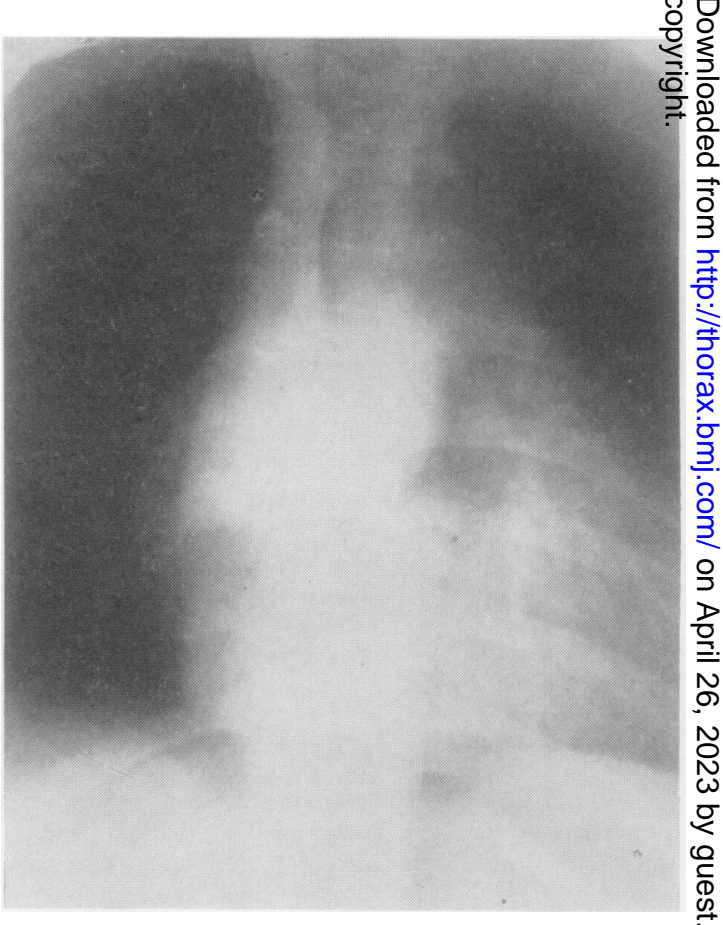

FIG. 3.-Angiocardiogram (frontal view). Note the smooth, rounded defect in the contrast medium filling corresponding to the right atrial tumour mass. 
The atrium was opened with a separate longitudinal incision after the circulation had been stopped, the total occlusive time being 5 minutes 50 seconds. The stalk of the tumour originated from the inferior part of the septum close to the posterior cusp of the tricuspid valve. The tumour was removed by division of its stalk.

The atrium was closed under saline solution by two rows of continuous mattress and over-andover sutures. The circulation was re-established by relaxing the tapes on the venae cavae and removing the clamps from the aorta and pulmonary artery. After injection of calcium chloride into the pulmonary artery the heart beats became strong and vigorous again. After that the pericardium was sutured. Closure of the thoracotomy was accomplished with separate drainage of both thoracic cavities.

Grossly the tumour resembled a ball measuring $4 \times 5 \times 5.5 \mathrm{~cm}$., glistening, with a smooth, slightly lobulated surface, weighing $49.8 \mathrm{~g}$. and of a yellowish white colour (Fig. 4). The cut surface was greyish with numerous islets of calcium deposits (Fig. 5). It was fragile, and when an attempt was made to grasp it with the jaws of a forceps it was easily crushed.

Histological examination of the specimen revealed a tumour mass with myxomatous and fibrotic changes and with frequent calcification.

The post-operative period was uneventful. After a considerable period of bed rest and restorative

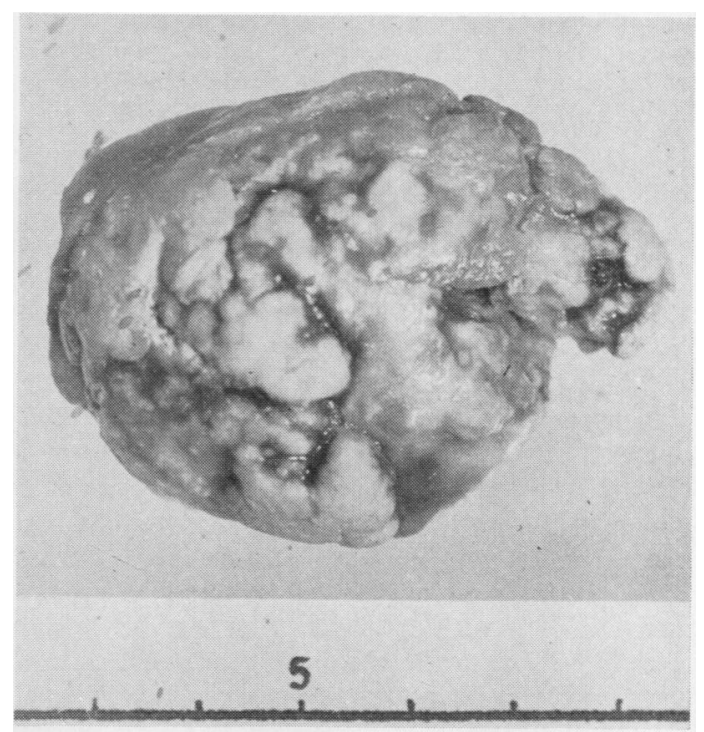

FIG. 4.-Gross appearance of tumour.

therapy the patient was discharged on November 20 , 1957 , well, without any complaints.

At the time of discharge there were still signs of tricuspid insufficiency, but the hepatomegaly had decreased and so had the expansile pulsation of the

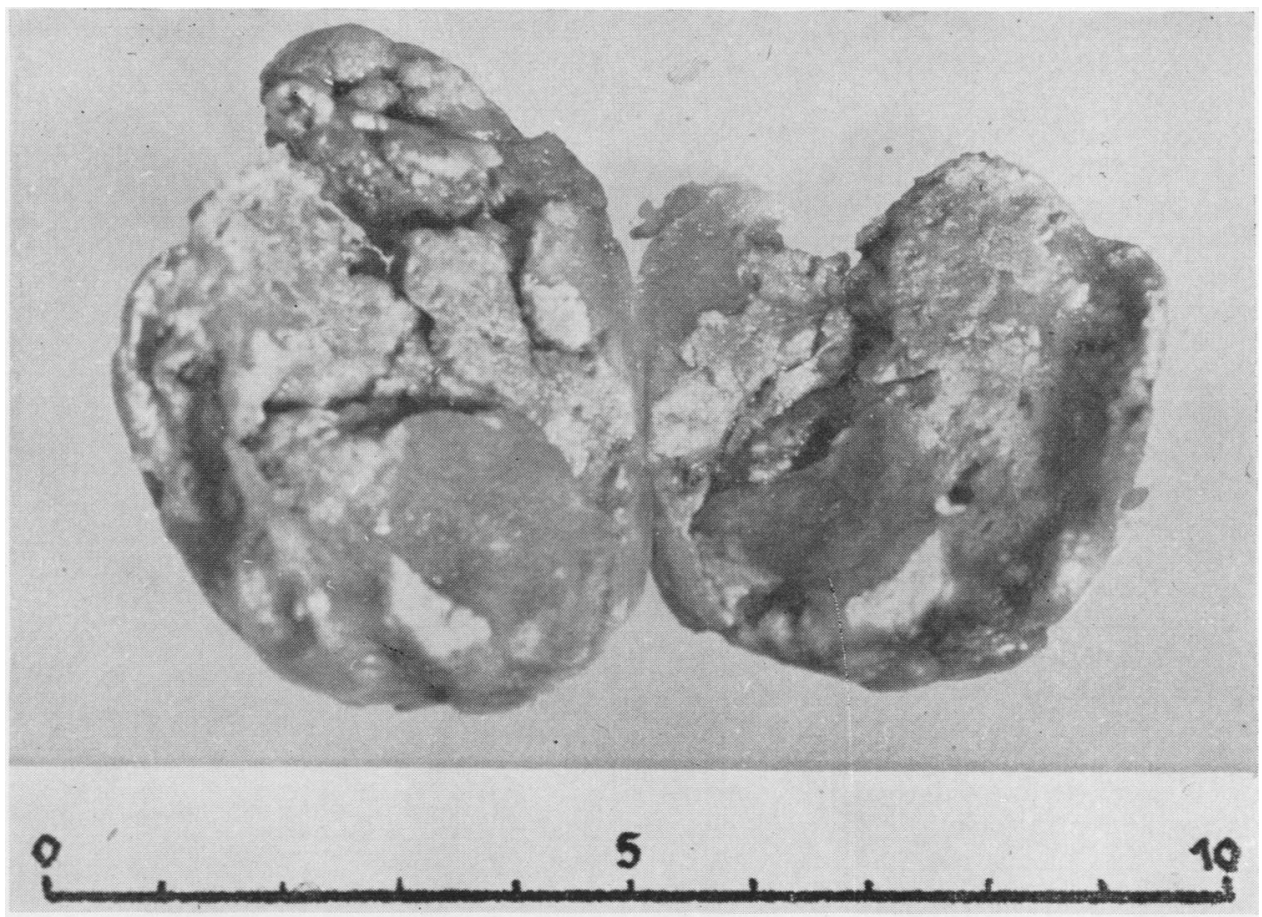

FIG. 5.-Gross specimen transected. 
jugular veins. The systolic murmur over the tricuspid valve remained unchanged. The signs of right ventricular enlargement on radiographic examination and electrocardiographic tracings persisted.

\section{Discussion}

Most primary cardiac tumours, according to Mahaim's monograph (1945) $25 \%$, and according to Prichard (1951) 50\%, are myxomata, described generally as polypoid formations with a stalk located in the atrial cavities of the heart. They are usually ball-shaped or pear-like with a finely lobulated surface arising on the atrial septum in the region of the fossa ovalis. According to Bailey (1955) they are four times more frequent in the left than in the right atrium. The growth of the tumour down to the tricuspid or mitral valve leads to partial obstruction of the left or the right venous orifices as it is driven to and fro by the current of blood (Mahaim, 1945 ; Prichard, 1951 ; Bailey, 1955). Disability and symptoms reminiscent of mitral or tricuspid stenosis with or without insufficiency are thus explained. The mobility of the tumour and its movements, depending on the position of the patient's body, account for the changing and inconstant auscultatory findings. The complete occlusion of a venous orifice by the tumour mass is followed by sudden disappearance of the pulse, unconsciousness, cardiac syncope, and sudden death (Bailey, 1955). Breaking off of small particles from the surface causes repeated and sometimes multiple arterial emboli in the case of a left, or pulmonary infarction in the case of a right, atrial tumour (Prichard, 1951).

The question is still under discussion whether these cardiac myxomata are tumours or thrombi with myxomatous changes (Mahaim, 1945). From his surgical experience with mitral stenosis, Bailey (1955) concludes that they are real tumours, as so far he has never found a thrombus on the atrial septum in a case of mitral stenosis.

Myxoma situated in the atrium has been diagnosed during life and pre-operatively only in a few cases. It has more frequently been found by chance at operation for mitral stenosis. Most attempts at removal have been unsuccessful and have ended with the death of the patient operated on. From the literature at our disposal we have only found four cases of myxomata successfully removed from the left atrium. These were a myxoma of the left atrium removed under extracorporeal circulation by Crafoord (1955) in a woman of 41 years of age; another myxoma of the left atrium was removed by Scannell, Brewster, and Bland (1956) in a man 33 years of age. Chin and Ross (1957) report a man of 25 years of age with a tumour of the left atrium removed under hypothermia, and finally Bahnson, Spencer, and Andrus (1957) removed a myxoma of the left atrium in a woman of 57 years of age under hypothermia and artificial circulation.

\section{SUMMARY}

The successful removal of a right atrial tumour in a girl 14 years old is described.

The tumour mass measured $4 \times 5 \times 5.5 \mathrm{~cm}$., and was hanging on a stalk from the inferior part of the right atrial septum close to the posterior cusp of the tricuspid valve.

Diagnosis of the tumour was made pre-operatively by radiological examination and angiocardiography.

\section{ADDENDUM}

Since this paper was submitted for publication we have operated on another tumour, this time in the left atrium.

The patient was a 51-year-old woman who had suffered from paroxysmal heart attacks for nearly three years. After a period of unsuccessful conserva tive treatment a tumour mass in the left atrial cavite was revealed by angiocardiography. The patient was referred to our clinic for surgical treatment as heop attacks were becoming more frequent and severe. On admission she was in rather poor general condition. The operation was performed on March 14, 1958 Using extracorporeal circulation we were successful in excising a tumour of oval form measuring $8 \times 5.2 \times 2.5 \mathrm{~cm}$., weighing $52.52 \mathrm{~g}$., and situated on the septal wall of the left atrium. Histological examination revealed a tumour with fibrous and myxomatous changes.

The patient's post-operative course was entirely uneventful and with her regaining strength she is now walking after more than one year's confinement to bed.

Operation was performed under hypothermia by surface cooling and on the opened heart with a total occlusion time of 5 minutes and 50 seconds.

Histology revealed a partially calcified myxomatous tumour with fibrous changes.

\section{REFERENCES}

Bahnson, H. T., Spencer, F. C., and Andrus, E. C. (1957). Ann Surg., 145, 915.

Bailey, C. P. (1955). Surgery of the Heart. Lea and Febiger, Philadelphia.

Bickford, B. J., Egan, M., and Bryce, A. G. (1956). Brit. J. Surg., 43, 514 .

Chin, E. F., and Ross, D. N. (1957). Brit. med. J., 1, 1447.

Chin, E. F., and Ross, D. N. (1957). Brit. med. J., 1, 1447. posium on Cardiovascular Surgery, p. 202.

Mahaim, I. (1945). Les tumeurs et les polypes du coeur. Masson, Paris.

Prichard, R. W. (1951). A.M.A. Arch. Path., 51, 98.

Scannell, J. G., Brewster, W. R., Jr., and Bland, E. F. (1956). New Engl.J. Med., 254, 601, cit. J. Amer. med. Ass. (1956), 161, 757. 